\section{Myocardial injury in patients with COVID-19}

In patients with coronavirus disease 2019 (COVID-19), myocardial injury is prevalent and is associated with an adverse prognosis and increased mortality. This finding comes from two retrospective studies involving cohorts of patients from New York, USA, and Sichuan, China.

A total of 2,736 patients with laboratoryconfirmed COVID-19 were admitted to one of five hospitals in the Mount Sinai Health System in New York between 27 February and 12 April 2020 and had their plasma level of troponin I measured within $24 \mathrm{~h}$ of hospital admission. Overall, $36 \%$ of patients had a troponin I level greater than the normal level (defined as $30 \mathrm{pg} / \mathrm{ml}$ ), and elevated troponin I levels were associated with a higher prevalence of cardiovascular disease. Mildly elevated troponin I levels $(30-90 \mathrm{pg} / \mathrm{ml})$, indicative of small amounts of myocardial injury, were associated with an increased risk of death (HR $1.75,95 \% \mathrm{Cl}$ 1.37-2.24, $P<0.001)$. This risk was further increased (HR 3.03, 95\% Cl 2.42-3.80, $P<0.001)$ in patients with a marked elevation in troponin I levels ( $>90 \mathrm{pg} / \mathrm{ml})$.

Similarly, in a study of 101 patients admitted to one of two designated COVID-19 treatment centres in Sichuan between 16 January and 10 March 2020, $15.8 \%$ had a plasma troponin I level greater than the normal upper limit $(14 \mathrm{pg} / \mathrm{ml})$, indicative of acute myocardial injury. These patients had a higher prevalence of pre-existing cardiovascular disease and were more likely to require admission to an intensive care unit and mechanical ventilation.

Hypothesized mechanisms of myocardial injury in patients with COVID-19 include myocarditis related to the SARS-CoV-2 infection, systemic release of inflammatory cytokines, coronary microvascular damage from thrombosis, direct entry of the virus into myocardial cells via ACE2 receptors, hypoxaemia and acute coronary syndrome owing to destabilized atheromas.

ORIGINAL ARTICLES Lala, A. et al. Prevalence and impact of myocardial injury in patients hospitalized with COVID-19 infection. J. Am. Coll. Cardiol. https://doi.org/10.1016/ j.jacc.2020.06.007 (2020) |Wei, J.-F. et al. Acute myocardial injury is common in patients with covid-19 and impairs their prognosis. Heart https://doi.org/10.1136/heartjnt-2020317007 (2020)

\title{
Resterilized pacemakers and defibrillators not associated with increased infection
}

Cardiac devices that are harvested postmortem, resterilized and reused are not associated with an increased risk of infection or device-related death compared with new devices, according to a new study in NEJM.

Patients living in low-income countries have limited access to life-saving cardiac devices. To address this issue, from 1983, the Montreal Heart Institute in Canada began sending pacemakers and implantable cardioverter-defibrillators (ICDs) that had been extracted post-mortem and resterilized to under-resourced countries and, in 2003, started to record outcomes of their device reuse programme in a prospective registry. These data were used to determine whether recycled devices were associated with increased infection or device-related death compared with new devices.

In total, 1,051 patients from the Dominican Republic, Guatemala, Honduras and Mexico who received recycled pacemakers or ICDs were included in the analysis and were matched in a 1:3 ratio to 3,153 controls from Canada who had a new device implanted. During the 2-year follow-up period, 21 infections $(2.0 \%)$ occurred in patients who received a reused device compared with 38 infections (1.2\%) in patients who received a new device (HR 1.66, 95\% CI 0.97-2.83, $P=0.06$ ). The only factor that was significantly associated with a lower risk of infection was younger age (HR 0.98 per year of age, 95\% CI 0.97-0.99). No device-related deaths occurred in either group.

"The 95\% confidence interval for the hazard ratio in the primary competing-risk analysis ranged from 0.97 to 2.83 , which suggests that, in a larger study, the difference between groups might have been statistically significant," caution the investigators. Nevertheless, they acknowledge that the absolute difference in risk between the two groups was very low $(<1$ percentage point).

Karina Huynh

ORIGINAL ARTICLE Khairy, T. F. et al. Infections associated with resterilized pacemakers and defibrillators. N. Engl.J. Med. 382, 1823-1831 (2020)

\section{ATHEROSCLEROSIS}

\section{Macrophage mimetics to target atherosclerosis}

A new biomimetic drug delivery system consisting of nanoparticles that are coated with macrophage membrane and responsive to reactive oxygen species (ROS) enables targeted pharmacotherapy for atherosclerosis in mice while also suppressing local inflammation by sequestering inflammatory factors.

Given that inflammation and ROS overproduction are hallmarks of atherosclerosis, Gao and colleagues hypothesized that this novel drug delivery system might provide high targeting efficiency and therapeutic efficacy. The macrophage membrane would enable the nanoparticles to evade clearance by the reticuloendothelial system and would target delivery to inflammatory sites, and the ROS responsiveness would enable specific drug release owing to the high local levels of ROS.

The researchers generated nanoparticles with a compound that degrades in the presence of $\mathrm{H}_{2} \mathrm{O}_{2}$ (thereby releasing the payload), loaded them with atorvastatin and coated them with cell membrane derived from mouse macrophages. The loaded biomimetic nanoparticles reduced inflammation and foam cell formation in vitro. These anti-inflammatory effects were mediated by atorvastatin and by receptors present on the macrophage membrane, which bound and sequestered pro-inflammatory cytokines and chemokines. In a mouse model of atherosclerosis, the loaded biomimetic nanoparticles had high targeting efficiency and showed targeted drug release to atherosclerotic plaques, leading to reduced plaque inflammation and decreased plaque burden. The cell-membrane system had better therapeutic efficacy than a live-cell carrier consisting of ROS-responsive nanoparticles internalized in macrophages, even though the live-cell system had a higher accumulation rate in the plaques. Given that this macrophage-biomimetic delivery system has inflammation tropism without the need for specific targeting molecules, this strategy might hold promise for other inflammatory diseases.

Irene Fernández-Ruiz

ORIGINAL ARTICLE Gao, C. et al. Treatment of atherosclerosis by macrophage-biomimetic nanoparticles via targeted pharmacotherapy and sequestration of proinflammatory cytokines. Nat. Commun. 11, 2622 (2020) 